\title{
Cerebellum and basal ganglia interaction essential in RDP
}

Dysfunctional interactions between the cerebellum and the basal ganglia are a key factor in the underlying pathophysiology of rapid-onset dystonia-parkinsonism (RDP), according to a new study. The root cause of this movement disorder seems to be aberrant activity initiating in the cerebellum, which then alters the function of the basal ganglia to cause dystonia. "Until now, the basal ganglia had been thought to be the only brain location whose dysfunction causes dystonia," says senior author Kamran Khodakhah (Albert Einstein College of Medicine, New York, USA), "yet literally hundreds of patient case reports implicated other structures, including the cerebellum."

RDP is characterized by an abrupt appearance of severe dystonic and parkinsonian symptoms after a stressful event (psychological and/or physical). Although mutations in the $\alpha 3$ isoform of $\mathrm{Na}^{+}-\mathrm{K}^{+}$-ATPase are known to cause the disease, the underlying mechanisms are poorly understood. Efforts to elucidate these mechanisms are hampered by the fact that genetic animal models often do not closely resemble the pathophysiology of the human disease-no current genetic RDP mouse model displays dystonia or dyskinesia.

\section{4 ...the basal ganglia had} been thought to be the only brain location whose dysfunction causes dystonia... 77

Khodakhah and colleagues developed a pharmacological mouse model of RDP that mimicked the human symptoms of the disease-dystonia, rigidity, tremor and akinesia-by selectively blocking $\mathrm{Na}^{+}-\mathrm{K}^{+}$ATPase with ouabain. Targeted perfusion of ouabain into the basal ganglia or cerebellum revealed that both structures were involved in RDP. Importantly, the stress-induced aspects of RDP were only replicated when the cerebellum and basal ganglia were perfused with ouabain simultanenously. Notably, disruption of the disynaptic connections between the cerebellum and basal ganglia (via the thalamic centrolateral nucleus) alleviated dystonia; off-target electrical lesioning in the RDP mouse model did not improve motor symptoms.

"The cerebellum can be the original site of dysfunction for dystonia," says Khodakhah, who credits the Hess-Jinnah research group (Emory University, USA) for "propagating the idea that the cerebellum can cause dystonia".

Further research into how cerebellar dysfunction alters brain activity is now planned and could lead to crucial insights into the pathogenesis of RDP and other (non-RDP) dystonias, as well as new pharmacological treatments or rational therapeutic approaches for RDP. One such dystonia therapy currently being explored by the study authors is optogenetic stimulation or inhibition of the affected brain regions.

\section{Katrina Ray}

Original article Calderon, D. P. et al.The neural substrates of rapid-onset Dystonia-Parkinsonism. Nat. Neurosci. 14 357-365 (2011) 